\title{
How NOT to create a digital media scholarship platform: the history of the
}

\section{Sophie 2.0 project}

Jasmine S. Kirby ${ }^{1}$

\begin{abstract}
Since the mid-2000s digital platforms have emerged to take advantage of the capabilities of new technology to incorporate media content, tell nonlinear stories, and reinvent the book for the 21st century. Sophie 1.0, from the University of Southern California, the Institute for the Future of the Book (IFB), and computer scientists based in Europe, was an attempt to create a multimedia editing, reading, and publishing platform. Sophie 2.0 was an international collaboration between the University of Southern California and Astea Solutions in Bulgaria to rewrite Sophie 1.0 in the Java programming language. This research will explore how the Sophie 2.0 project was unable to become a viable and well-maintained open source product despite receiving over a million dollars in funding from the Mellon Foundation. Problems included the technological difficulty of creating an easy-to-use but completely customizable open source multimedia e-publishing platform, which was also compounded by competing visions over what this project was to be. Stakeholders did not demand a deliverable that actually worked. Funders seemed willing to overlook weaknesses in early releases for a more encompassing, if impractical, project. The computer scientists wanted to add the most features possible, while the IFB and USC Institute for Multimedia Literacy focused on creating a product based on the values of a future they hoped to create. Understanding what went wrong with Sophie 2.0 can help us understand how to create better digital media scholarship tools and to start much-needed discussions about failure in the digital humanities.
\end{abstract}

\section{Keywords}

Sophie, Digital Humanities, Failure, USC, Institute for the Future of the Book, Astea Solutions,

\section{Introduction}

We don't talk enough about failure in the digital humanities. This is a problem because as information professionals we are expected to find, understand, and explain digital tools for scholarship that we want our users to be able to access for eternity. In the field of librarianship and in higher education, there is a particular emphasis on supporting free and open source platforms, since these are thought to embody the values of openness, transparency, and continued access that we promote and aim to achieve. However, it can be very hard to tell which open source projects are going to succeed and which will flop. Studying failed projects can give us guidance on what to look for when choosing digital humanities tools for our own research and the research of our library users. While some of these failures are products of their unique time and place, they also speak to many dangers in software development that apply to other projects. Although it received over a million dollars from the Mellon Foundation and others, Sophie 2.0, an update on the Institute for the Future of the Book's Sophie 1.0 multimedia e-book reading and authoring platform from the University of Southern California and

1/16 Kirby, Jasmine S. (2018) How NOT to create a digital media scholarship platform: the history of the Sophie 2.0 project, IASSIST Quarterly 42 (4), pp. 1-16. DOI: https://doi.org/10.29173/iq926 
Astea Solutions never became a viable digital humanities and media scholarship platform. This paper will explore factors that contributed to the failure of the Sophie 2.0 project.

\section{Situational context}

There is limited scholarship available about failed digital humanities projects and the failure of the Sophie project in particular. This research will draw from "Whatever Happened to Project Bamboo?" an article written in 2014, where Quinn Dombroski, a member of the staff of an institution participating in the digital infrastructure initiative Project Bamboo, discusses the issues that resulted in the failure of that project. There is not much critical information about the Sophie project in particular. In 2010, Dan Visel, from the Institute for the Future of the Book (IFB), who was in charge of software development for Sophie, interviewed the institute's founder, Bob Stein, discussed Stein's influential role in the history of computers, and briefly mentioned the Sophie project. There is a 2011 interview of Bob Stein that while focused on his accomplishments and those of Institute for the Future of the Book, does include a section on problems with Sophie 1.0 and Sophie 2.0. Both of these published interviews help us understand a lot of the context and thinking behind the Sophie project and what it was building on, blaming its failure on too much ambition and a lack of funding, but do not go into enough detail into the reasons that the Sophie project failed. Beyond these interviews, there is so little objective information available about Sophie and its end that there is still confusion as to whether it is a viable platform. For example, as recently as January 2017, there was an article in the magazine Computers in Libraries about digital humanities still promoting Sophie 2.0, although the author, Nancy K. Herther, an academic librarian, acknowledged the lack of updates, but mentioned looking forward to what the project is said to bring (Herther, 2017).

I want to emphasize the importance of seeing computer applications in the greater context of history of media and history of science and technology to contrast how narratives about new software tend to focus on how they are unprecedented and unique to the $21^{\text {st }}$ century. I build on Ballatore and Natale's work on the cultural implications of what they call 'the myth of the death of the book' and agree that "Such prophecies, however, are revealing of the way societies regard media as vehicles for change - precisely because they are embedded in the idea of the future." (Ballatore and Natale, 2016). I agree with Evgeny Morozov, in his work To Save Everything Click Here that the Internet needs to be studied not as a "McLuhanesque 'medium" or the bringer of a unique epoch in human history and instead placed in a greater context (Morozov, 2013). Another article that inspired the way this research is framed is "Listening to Pictures" by Katie Day Good, a media scholar. This article discusses the history of Radio Photologues, a combined radio program and photogravure section in the Chicago Daily News, and explores the context and significance of this unique media product. Similarly, I hope to place Sophie in a longer history of mixed media forms. I want to illustrate the important lessons that digital humanists can learn from looking past our cultural myths about media and instead examine the actual complicated messy history of how new media emerge and change over time.

\section{A brief history of the future of reading}

The history of Sophie starts in 1981, with publisher Bob Stein encountering "electronic text that might be readable" and an early demonstration of video embedded in hypertext and "...realized at that point 
that the book of the future wasn't going to be limited to text and figures; we were going to be able to have audio and video on the page"(Stein, 2008). Sophie 2.0 is but a chapter in the long and troubled history of e-books and e-book platforms. While the idea of the e-book can be found earlier in computer history, in their article about the history of the idea of e-books resulting in the end of the print book, Ballatore and Natale describe how "The development of the actual idea of the e-book is principally attributed to Andries van Dam, who coined the term working on a hypertext system in 1967, and Michael Hart, who founded Project Gutenberg in 1971" and describe how early attempts at e-books were hindered by the size and limits of computers of the 60 s and 70s (Ballatore and Natale, 2016). In his article "A Call to Embrace Social Reading in Higher Education" business professor Matthew Dean describes how "In a hard-copy version of a book, one can highlight sentences, annotate in the margins, bookmark important pages, keep the book in a revered spot on a bookshelf, loan it to friends, discuss your favorite parts with others who have read the book, etc." (Dean, 2016). E-book creators face the challenge of either replicating the beloved features of a book in a digital environment or creating something better.

Bob Stein figures prominently in both the history of e-books and the story of Sophie. Not many other people had the vision to see past the limits of the technology at the time and believe in the possibilities that new technology offered for changing the way we learn. Before even the original Sophie, Bob Stein would try to build a multimedia editing platform of one form or another at least twice. While running the Voyager multimedia electronic publishing company, Bob Stein oversaw the creation of the Expanded Books toolkit, a software that allowed educators to create and edit their own editions of books on floppy disks (Rüger et al., 2008). Stein is described in a 1996 profile of his Voyager company as "the most far-out digital publishing visionary in the new world or the least effective businessman alive - or both" and that "Many people who work for Stein mention his tremendous intellectual passion and enthusiasm - and an almost equal number cite his short attention span and complete disregard for detail" (Virshup, 1996). That being said, his laserdisc film collection offering a second audio track of commentary was the first of its kind and changed the way we study film, and Voyager offered a variety of multimedia educational products including, most famously, the interactive primary source collection Who Built America CD-ROM (Visel, 2018).

Stein of course was not the only one interested creating a platform allowing users to create multimedia products in the 1980s and 1990s. In 1987 Apple created the HyperCard application that allowed users to create documents that "could contain images, sounds, and movies; the author could add controls via a simple language called HyperScript" however, "Apple killed the project in 2000..." (Stein and Visel, 2010). The story of Hypercard shows that similar programs to allow non-programmers to create interactive multimedia documents had been tried before in the closed source world with limited success. Moreover, Ballatore and Natale in their discussion of the history of e-books indicate how early attempts at e-readers during the 1990s were considered a failed technology and did not survive the dot-com burst (Ballatore and Natale, 2016). While of course the technology was much more limited, it does show that multimedia e-books and e-book creators were never popular.

Stein also led The Night Kitchen company that created TK3, the predecessor software to Sophie that was a closed source product (Rüger et al., 2008). TK3 was probably the most successful of all of Stein's efforts to create a multimedia editor and reading platform. For example, according to Visel, while no one ever put their thesis in Sophie 1.0 or Sophie 2.0., Virginia Kuhn, a media scholar, did her thesis in

3/16 Kirby, Jasmine S. (2018) How NOT to create a digital media scholarship platform: the history of the Sophie 2.0 project, IASSIST Quarterly 42 (4), pp. 1-16. DOI: https://doi.org/10.29173/iq926 
TK3 and got it accepted which was a major development for e-books since it was the first born digital doctorate (Visel, 2018). It is clear that there was some acceptance of the use of TK3 for scholarship purposes. In an interview he did with Visel, Stein describes the end of TK3 with his refusal to get rid of the Macintosh version in order to get funding from Microsoft to market the software and then ultimately abandoning the project (Stein and Visel, 2010). The end of TK3 speaks to Stein's dedication to creating software available for users on all types of platforms and wanting to reach the broadest audience possible. Telling off the largest software company in the world at the time also demonstrates that Stein had a specific vision of how software should work and be available to people. This also is part of a larger pattern of Stein not letting things like profit or feasibility get in the way of his vision.

Despite the previous limited adoption of multimedia e-book creators, Stein was given the opportunity to create an open source version of TK3. Specifically, "The Mellon Foundation approached some of the TK3 team and asked them to build a new multimedia authoring program which would extend TK3 by enabling time-based events and make it able to live on the network. That became Sophie" (Rüger et al., 2008). In their conference paper "Sophie: The Future of Reading" the creators of what would become Sophie 1.0 describe their project as "With Sophie we are tackling the long standing issues as keeping documents and their media accessible for a long time (the 200 year problem) and making electronic books living documents that capture and reflect the readers' interactions and comments (the annotating problem)" (Rüger et al., 2008). Using funding acquired from the Mellon and the MacArthur foundations, Stein founded the Institute for the Future of the Book in 2004, a think tank that continued his longstanding affiliation with USC but was based in New York (Stein and Visel, 2010). Why did nonprofits, most notably the Mellon Foundation, ignore the unpopularity and unprofitability of earlier efforts to create multimedia e-book platforms and support this venture?

\section{Issues inherited from the original Sophie}

The Sophie project was funded by the Andrew Mellon Foundation as part of their Research in Information Technology program (Mellon/RIT). This program sponsored projects that would create "community-source software" and "service-oriented architecture" where universities would work together to develop shared platforms that would reduce the amount of redundant software applications and be open source so people wouldn't be stuck with certain vendors (Fuchs, 2008). In other words, creating big applications that serve lots of functions and can be customized to the unique needs of different institutions to replace lots of small applications (Fuchs, 2008). In her article on Project Bamboo, another failed initiative sponsored by this grant, Dombroski argues that the main purpose of this program was to make digital tools that everyone can use instead of everyone building their own tools for their own projects (Dombrowski, 2014). This goal of creating large platforms to combine overlapping software needs explains why a grant program would be interested in TK3 with its ambitious goal of allowing for multimedia e-books and previous use for digital scholarship. In a 2007 conference paper from the Forum for the Future of Higher Education, Visel describes the advantages of Sophie with: "More and more students are taught to make presentations with PowerPoint, a limiting program that, as Edward Tufte has pointed out, encourages gimmicky special effects at the expense of coherent thinking...Sophie treats all media equally: if adding a slide show would be helpful to the primarily written report, the student can add the slide show to the page it is intended to illustrate without having to switch from Word to PowerPoint" (Stein and Visel, 2007). This

4/16 Kirby, Jasmine S. (2018) How NOT to create a digital media scholarship platform: the history of the Sophie 2.0 project, IASSIST Quarterly 42 (4), pp. 1-16. DOI: https://doi.org/10.29173/iq926 
is the idea of creating tools around the work that universities are doing rather than being locked into the functionality provided by proprietary software such as Word and PowerPoint that are more oriented towards the needs of corporations than universities. Stein and Visel also write "Sophie's aim is to democratize the world of multimedia by making it possible for individuals and small nonprofits to express themselves via compelling multimedia books" (Stein and Visel, 2007). Moreover, an open source version of TK3 would also help Mellon's overall mission of keeping the humanities relevant in the $21^{\text {st }}$ century by letting scholars incorporate multimedia.

In interviews about what happened, over-ambition and lack of funding are blamed for the failure of Sophie and other digital humanities initiatives. This issue of creating and releasing ambitious and difficult to complete software was compounded by the restructuring of the Mellon Foundation in December 2009, when RIT became part of Scholarly Communications, resulting in a new group of people to work with, different goals, and less money available (Dombrowski, 2014). All of this was made even worse by the 2008 financial crisis and a shifting of priorities at universities away from futuristic long-term projects to software that addressed local needs (Dombrowski, 2014). We now know that the most successful open source projects have paid staff working to keep them up to date, which was not the case with Sophie which had money to create essentially a demo product but could not get funding to keep it going (Visel, 2018).

It's also true that there was a dramatic staffing change between the first and second editions of Sophie. It's likely that Sophie was facing similar issues to the case of Project Bamboo where "These staffing changes led to a loss of organization memory, which had particularly negative consequences for the message and tone of the project's communication with scholarly communities" (Dombrowski, 2014). It is still possible to access forum postings from 2008 on Slashdot where an alleged former programmer from Sophie 1.0 argues that their work has been stolen by USC and Astea Solutions ("How To Kill an Open Source Project With New Funding," 2008). In addition, coordinating a project between USC in Los Angeles, the Institute for the Future of the Book in New York, and Astea Solutions in Bulgaria with the technology at the time was not the easiest feat to accomplish. A Sophie user described that the geographic distance meant that it could be very hard to get tech support; and that they imagined getting support would be impossible if you were not friends with one of the people behind the project who could contact the programmers in Bulgaria directly. ${ }^{2}$ On the website for Sophie saved in the Internet Archive, even the most updated version from June $19^{\text {th }} 2015$, the link for Technical Support is a mail:to to Daniel Visel ("Sophie," 2015).

Sophie 2.0, the update of Sophie 1.0 in Java, would've been difficult regardless but on top of everything else Sophie 1.0 was a very unique software with many incompatible parts and ideas. To start, due to the influence of computing pioneer Allen Kay and a desire to deal with the problem of digital preservation, the original Sophie was written in Smalltalk (Visel, 2018). However, Smalltalk was a very academic programming language that had mostly been used by the Swiss banking system, which meant that there was no video player or text editing (Visel, 2018). Smalltalk had the advantages of being device independent, for example, you can still run Smalltalk programs from the 1970s (Visel, 2018). People want to be able to access their academic work in the future. For example, although it was accepted and serves as part of the basis of her academic career, to look at Virginia Kuhn's thesis in TK3 these days you'd need to use a Mac emulator running on a Mac (Visel, 2018). This is unacceptable for widespread use in research and writing contexts where people need to be able to

5/16 Kirby, Jasmine S. (2018) How NOT to create a digital media scholarship platform: the history of the Sophie 2.0 project, IASSIST Quarterly 42 (4), pp. 1-16. DOI: https://doi.org/10.29173/iq926 
access stable versions of their scholarship for years as they build up their careers. Creating a multimedia editing platform from a programming language mostly used for banking is a very difficult task by itself. Smalltalk was so obscure that everything had to be created from scratch and the programmers couldn't build on other people's code (Visel, 2018). Sophie 1.0 was supposed to be built to last. What's more, this meant that not only were creators of Sophie 2.0 unable to use any of the original code but they also could not use any of the workflows that came out of the Sophie 1.0 project since it was a very different kind of project. Visel summarized the issues that came from using Smalltalk for Sophie 1.0 with, "It was a really good idea but a really hard idea" (Visel, 2018).

Looking at Sophie and its various iterations it becomes increasingly clear that the focus was on building the tool with the most features and not something that actually could be sustained or even worked. The computer scientists who programmed Sophie 1.0 described how "[e]ven though a system may work correctly, it may still fail in the field of user experience and usability if it does not embrace suitable concepts to implement and to offer the possible very large number of expected features" (Holz et al., 2009). In a 2011 interview, in a discussion on "Sophie and Software Development" Stein mentions that "As a publisher, I learned to live with the "get-it-right-the-first-time" reality of print, but it's a completely wrong model for software development in the era of the digital network, where the goal is to get out a good-enough first version and then iterate and improve as fast and as often as you can" (Gold, 2011). In that way, Sophie faced many of the same issues as Project Bamboo. "However, the infrastructure was architected in such a way that made it difficult to complete and release standalone components that could be tested and used while other parts were incomplete...The extensive development time required for infrastructure components without successfully fulfilled real needs..." (Dombrowski, 2014). It is clear that the creators of Sophie wanted to build a complete product, which also explains why this project took such a long time to create anything and needed so much money.

The innovation and uniqueness aspect of Sophie 1.0 also went into the user interface and experience. In a review of the Sophie 1.0 alpha release, early adopter, tech blogger James Bridle, writes "It's clearly inspired by existing rich media applications such as Flash, but it's [sic.] target users - the technologically unskilled - don't use such applications. How are they supposed to get their heads around concepts such as 'flows,' 'timelines' and different server versions? And if they do get that, why aren't they using the existing apps? It's all very disappointing, and I think if: book ${ }^{3}$ [sic.] know it, which is why they haven't supported or trumpeted this release in any way" (Bridle, 2007). Even as early as the alpha release, before the global recession limited the possible support from Mellon and academic institutions, there were issues with the design of the software itself. Having to build everything from the ground up also likely resulted in a user interface that one Sophie 1.0 user described as "outdated" and "like running an emulator." ${ }^{4}$ Visel, who wrote the documentation, described the software as "deeply, deeply confusing." On the Sophie 2.0 developer site, there is still a page that reads "Sophie Wishlist (This is a list based on discussion with Bob Stein and the Institute for the Future of the Book's Sophie users as well as looking through Mantis feature requests)." Items on the wish list, which were things that the developers openly admitted probably wouldn't be fixed, included not having a usable Windows file format, no way to delete embedded books, and "Sophie 1.0 doesn't handle saves well when the book has been moved to another location - this is something that people tend to do a lot (esp. on Macs, I think) and there have been a lot of crashed books because of this. This needs to be handled more gracefully" (Visel, 2008). Instead of focusing on a less ambitious but functional project, 
Sophie 2.0 traded the lessons and benefits of Smalltalk in order to build a product that would be able to be maintained by a dedicated open source community.

\section{And then, it got worse}

In a forum post on Slashdot, a user claiming to be Elizabeth Daley of the University of Southern California and principal investigator of the Sophie 2.0 project, explained that part of the reason that Sophie never took off was that institutions could not properly support a software written in Smalltalk, and that changing the language to something more commonly used would help reach the goal of creating a community to help develop it, as was required to get another grant from Mellon ("How To Kill an Open Source Project With New Funding", 2008). Changing the language it was coded in to Java did not change the fact that this was an open source software that did not particularly benefit open source users. Sophie was a multimedia editing platform meant for people who could not code, such as publishers and academics. This discussion on Slashdot was one of the few examples of any outreach towards programmers and information technology specialists for building the open source community to maintain the software. It is unclear who was ultimately supposed to keep the software up to date. The targeted audience of people who cannot code are the same people who are unable to fix or perhaps even articulate issues that inevitably come up in such a complicated piece of software in an environment of constant and rapid technological change. Visel mentioned at the time that the Mellon Foundation seemed to believe that if a digital technology was released as an open source software a community would build up around it to update it and add new features (Visel, 2018). While there have been some historical examples of this, such as the Linux operating system, these tend to be technical software aimed at technical users who have some coding experience already. Moreover, looking at the Sophie 2.0 developer site, it would be very hard to actually get involved in this project if a person wasn't in Bulgaria and working for or in some way affiliated with Astea Solutions since it appears that potential contributors had to get approval from the main team to do anything ("Ipandeff", 2009). Even if contributors were interested in joining this project, the developer's site is confusing, and it would be difficult coordinating time zones for contributors who were not based in Europe, such as IT people affiliated with USC.

What's more, Sophie 2.0 maintained the Sophie 1.0 team's practice of releasing unfinished alpha software to unsuspecting users. A Sophie 2.0 user described how "It was super unstable, crashed, and no clear rhyme or reason to why it kept crashing... In the process of building the Sophie book things would be gone and not retrievable not ever getting to the point of being finished." ${ }^{5}$ The Sophie 2.0 team never released a reliable deliverable; it was always up to the user to imagine what could have been while dealing with the reality of the limitations of the software in front of them. Instead of creating a smaller project that could do one thing well, they created a project that could kind of do a variety of things but was buggy, prone to breaking, and rarely up to date. This is neither appealing to the intended end users who are people who do not know how to code or the intended open source community who can't see what the overall vision of the project was supposed to be based on the code that was released. 


\section{Who was the audience?}

Another aspect that played a major role in its downfall was that Sophie lacked a clear target audience. Like many projects relying exclusively on grant funding, the target audience for Sophie changed based on who they were talking to. For example, a conference paper about Sophie 1.0 from the Forum on the Future of Higher Education mentions "While Sophie can be used in many settings it is aimed squarely at the world of education" (Stein and Visel, 2007). There is a promotional video put out by USC IML focused on using Sophie 2.0 for journalism (ArtsJ09, 2009). The grant proposals and papers list even more possible uses. For example, SmartBook, a project proposing to expand the functionality of Sophie 2.0, received funding from the Bulgarian National Science Foundation, and argued that an expanded Sophie 2.0 could indirectly benefit the way that science was published and discussed (Koychev et al., 2013).There was very little that Sophie could not be used for in some way or another.

One exception of course comes from The Transliteracies journal which pointed to the fact that Sophie was not being used for narrative storytelling and only for educational purposes as another weakness of the platform (Hudson, 2008). Being exclusively for education and not entertainment makes it similar to the Radio Photologues of the Daily News, where the ultimate problem of that platform was not that it was worse than other forms of media during the 1920s but that what people used it for didn't use narrative structures (Good, 2017). It is true that in the mid-2000s mainstream publishing companies were experimenting with releasing mixed media books. The idea was to reach audiences who were more used to, as one publisher explained, "three-minute YouTube videos and using social networks," with experiments such as including videos in electronic books that could be read online or on Apple devices or a website where readers could discuss the events in a book and possibly have their comments incorporated into later books (Rich, 2009). However, it seems Sophie did not reach out to these markets in any meaningful way until a report from 2011. Specifically, the research into potential users from the Bulgarian software developers focused primarily on reaching out to publishing companies. This research was done as a requirement of a Mellon grant and they did marketing research on publishing companies with a survey that didn't have a high response rate ("Sophie 2.0: From Projects to Publishing Initiative Two, Part One Marketing Analysis Results," 2011). Building digital humanities tools that tell narratives is important since that is the dominant form of how we relate to each other and how we currently communicate information. In trying to build a platform that was usable to everyone they created a product that was not particularly useful to anyone.

\section{Was Sophie ahead of its time?}

Sophie users often lament that the software was ahead of its time. But the real problem was that it was built for a future that would never be. Looking at conference presentations and interviews given by some of the minds behind Sophie 1.0 and Sophie 2.0 it becomes very clear that they, like many people during the first decade of the 2000s, subscribed to a narrative of technological progress and the idea of computing technology completely changing the way we live our lives. For example, a paper by the Bulgarian computer scientists who took over the Sophie 2.0 project begins with "The information technologies available today have made possible the advent of the e-book that overcomes a number of weaknesses of the classic scroll described so well by Socrates 24 centuries ago" (Koychev et al., 2013). The scientists see themselves as solving a problem that books themselves were not able

8/16 Kirby, Jasmine S. (2018) How NOT to create a digital media scholarship platform: the history of the Sophie 2.0 project, IASSIST Quarterly 42 (4), pp. 1-16. DOI: https://doi.org/10.29173/iq926 
to solve and ushering in a new era in the way that they believed print books did. The scientists building on the Sophie project see themselves as part of a long tradition of technological disruption and changing the world to a better place.

In their article "A Pedagogy for Original Synners" the authors, scholars from the Institute for Multimedia Literacy describe Sophie and similar efforts with "The limited range and noncommercial aspirations of such programs place emphasis on developing conceptual sophistication rather than final polish. We believe that this emphasis on process over product may allow students to pursue more experimental, concept-driven creative and critical production" (Anderson and Balsamo, 2008). This is a way of saying that they were less concerned about providing students with tools that actually worked than having tools that would encourage students to do a certain kind of creative work.

The main users of Sophie were educators preparing their students for a certain future they imagined. For example, the "Original Synners" pedagogy hoped to "address the learning needs of the born digital generation. 1. Open... 2. Hybrid... 3. Media rich..." (Anderson and Balsamo, 2008). The futuristic stance of the software is also demonstrated in the rare test uses for the software, $\mathrm{K}-12$ and undergraduate writing courses. For example, when describing using a Sophie 1.0 book for teaching an AP Spanish course, private school teacher Sol B. Gaitán describes how, "As a teacher of children and adolescents, I firmly believe I have the moral obligation to prepare them for the world they will be part of as adults" (Gaitán, 2011).

This also explains the way that this software dealt with issues of intellectual property, which is to say for the most part, it didn't .Perhaps this is because under the Fair Use doctrine in the US, copyrighted materials can be incorporated in educational materials under certain conditions. It is also possible that the neglect of intellectual property law could be what Barnett describes "[a]s part of the evolutionary history of e-books, the proliferation of pirated texts as digital files in the 1990s and 2000s created a network and market for the creation and consumption of digital texts. These were frequently circulated as Microsoft Word files, copied and pasted from OCR scans or typed by fans."(Barnett, 2015). But more importantly, Visel described in an interview how there was an expectation during the 1990s and 2000s that with the web information would be free and available online (Visel, 2018). This perhaps explains why in the paper for Sophie 1.0 the creators mention "Sophie to date does not deal with implement or enforce any $\mathrm{DRM}^{6 \mathrm{v}}$ related technologies, possibly making some local media resource unavailable for use within Sophie" (Rüger et al., 2008). Both Sophie 1.0 and Sophie 2.0 in their documentation and description deal very little with issues of intellectual property, despite being a software that is concerned with helping people create and preserve multimedia content. And it's not that people weren't thinking about intellectual property issues during the time. For example, a professor who used Sophie 2.0 for teaching a computers and writing course mentioned how learning about copyright and dealing with the fact that most film is still protected by copyright was a major part of an assignment of creating a digital edition in Sophie 2.0 (Bjork, 2012). It's clear that the few actual test case uses of Sophie 2.0 were dealing with copyright, even if the software itself did not.

As a software that dealt with both writing and multimedia, there was the constant question of whether Sophie was an aggregator or an authoring platform or both. "The Sophie server will provide a repository for all Sophie books that exist on a given network and will allow users to search Sophie books already created, as well as publish new books on it. The repository will also serve as a rich source 
of reusable content" (Stein and Visel, 2007). This reflects another idea from the time period that all new works would be combinations of old works and all merging into one big work as information became available and free online (Lanier, 2010). Sophie is an authoring, reading, and publishing platform for multimedia documents. While the creators were caught up in the view of a new culture, users on the ground were just confused. In Bridle's review of the alpha release of Sophie 1.0, there is a comment from someone identifying themselves as a developer from the Sophie 1.0 team who writes

"James, the reason Bob says 'assemble' and I say 'edit' is a philosophical difference in how we see Sophie... However, you look at it, the simple facts are that Sophie does (within the limits of bugs and available developer time) provide an editing tool for structured text, with searching, spellchecking, undo/redo, markup, annotation (for both author and reader, independently sharable by groups working on the same book) *as well as* assembly tools for other content formats. Comment by Tim Rowledge - April 12, 2007 @8:44 pm [sic.]" (Bridle, 2007). To which James Bridle, the author replied, "Tim - what I am suggesting is that 'philosophical difference' is not helping end users figure out what they are supposed to do with this software" (Bridle, 2007). This confusion over Sophie's purpose continued into Sophie 2.0 where "USC's support for Sophie has also included funding a week-long workshop for scholars in May 2008, during which Sophie's affordances were tested in practice in tandem with discussions regarding the ways in which Sophie transforms the traditional acts of scholarly reading and writing. Organized and led by the IML, the workshop raised several key conceptual issues. One of these centered on the tension between understanding Sophie as a compositional environment that sparks new forms of writing, in opposition to imagining Sophie to be an aggregator, or a space for gathering and displaying various texts and media objects" (Visel, 2008). The focus of the 2008 workshop is how Sophie 2.0 could be used to change scholarship, not how Sophie 2.0 fit with the scholarly practices at the time. It is clear that what the software was actually for was a question that remained unanswered throughout its entire history.

\section{A revolution in reading}

Before going into e-books, Stein worked as a leftist publisher for many years (Stein and Visel, 2010). The influence of leftist political thought played a role in his leadership both of his own companies and of the Institute for the Future of the Book. Sophie 2.0 continued the leftist visions of Bob Stein in particular with the way that the authorship feature is set up. Specifically, in a comparison of the strengths and weaknesses of various e-book platforms, one complaint about the authorship feature of Sophie 2.0 was "It is not possible to assign roles and planning activities when writing a book. All users can write at the same time, any place, and there is no way to block a section to avoid conflicts when writing nor it's [sic.] possible to know which section of the book was written by a particular user" (Ochoa et al., 2013). This is a structure of authorship without hierarchies where all authors are considered equal in both level of importance and in contribution regardless of amount. This complex rethinking is an idea of authorship that was promoted by the Institute for the Future of the Book. Specifically, 'An old-school author,' says Stein, 'is somebody whose commitment it is to engage with subject matter on behalf of future readers. A new school author is somebody whose commitment is to engage with readers in the context of subject matter... Authors are about to learn what musicians have already learned, which is, they're going to get paid to show up, whether it's at a speaking gig at 
a university or on a page of their book'" (Moyer, 2009). Although this was not the economic model that the world of writing operated on during the mid-2000s this was clearly the model that the Sophie 1.0 and Sophie 2.0 platforms were based on. Moreover, in the "What IF" article describing the Institute for the Future of the Book, the author discusses how "Adherents of the network book, though, such as online Wired magazine's Kevin Kelly, suggest a luminous future for reading as the number of available books rises with the creation of what is often called the 'universal library': 'Plans like Google's [to digitize out-of-copyright and out-of-print books] will allow all the books in the world to become a single liquid fabric of interconnected words and ideas" (Moyer, 2009). Sophie was built for a world where all information is free and available online. For example, in Sophie 2.0 "There is no statistical information to know how many users uploaded, downloaded, or liked a book" (Ochoa et al., 2013). Part of why there is so little information available about Sophie 1.0 and Sophie 2.0 was that there were no mechanisms in the platforms themselves for recording such information. This would have made it harder to justify their use by anyone since it would be hard to prove that the software was ever being used. There is a little bit of a ranking feature for individual users on the spinoff virtualbookclub platform with SophieServer with choices of "Good," "Ok," and "Bad" and public annotations (Hirschfeld et al., 2008). However, this was only on virtualbookclub and the people who actually used Sophie 2.0, such as the people reviewing e-book platforms for open educational content were unaware of this by the time they reviewed and these "Good" "Ok" and "Bad" rankings do not tell us very much about who is using these books (Ochoa et al., 2013).

Looking at Stein's writings from the Institute for the Future of the Book, he clearly felt a sense of responsibility for bringing about an inevitable techno-utopian vision of the world. For example, Stein describes an Institute for the Future of the Book experiment with commenting and annotation software with "Many of the earlier reviewers said the same thing: It is no longer the author speaking, it is now the book speaking" (Stein, 2008). This is an erasure of the individual from the experience of text and a promotion of a world where the group overrules the individual. The increased focus on social features of the platform and experiments at the Institute for the Future of the book in developing social networking capabilities show this dedication to a collective knowledge future where the individual was less important than the whole. After a talk in 2008 for serials librarians, Stein was asked about authors who may not want to have reader comments in their work or to change their work based on reader comments, and replied "I think that is a valid question you ask, but I would argue that over time what is going to emerge is a form of expression where artists will take for granted and want an intersection with the reader. The role of the reader and author is going to morph or merge in some way. I'm all for people doing things the way they want to do them, but I think things are going to change in the direction that I'm talking about" (Stein, 2008). Here is an example where Stein does not really give a satisfactory answer to a valid question but rather believes that the culture will inevitably change because of the change in technology. Therefore, the job of the creators of Sophie was to create the technology that would bring about the culture of the future rather than to build the technology for the culture that existed at the time. Sophie was more than just a software for creating interactive multimedia e-books that readers could annotate, it also was the responsibility of the Institute for the Future of the Book to wrestle with important questions of "what does it mean to be human in the age of the digital network" (Stein, 2008). During this same talk he warned "In fact, if we just cling to the past, then the techies who actually don't think about things are going to invent a future for us that we're really not going to like" (Stein, 2008). This is the idea of the responsibility of librarians 
and scholars to actively choose and shape what technology they use to share their work. Therefore, the marketing strategy for this product seemed to be that in a world where people have many options for how they express their work, people will choose the forms that bring about the vision of the world they want to create. This raises the question of whether Sophie was ever supposed to work or was it just a way to promote the values espoused by the Institute for the Future of the Book and the Institute for Multimedia Literacy at the University of Southern California.

\section{Conclusion}

Both Sophie and Sophie 2.0 fit into a greater history of using new technological advancements as a form of outreach and a way to showcase a world people want to exist. In her article describing the history of the Chicago Daily News radio and photogravure hybrid programming, Good discusses how "First, it is not clear exactly what kind of benefit these 'supplemental' extensions of the newspaper brought to the Daily News in terms of sales or readership. The Daily News approached both the citywide lecture series and the Radio Photologues as a public service that would boost the paper's image as a progressive, civic-minded source of information and cultural uplift"(Good, 2017). On the Sophie 2.0 "About Page" USC's role in the Sophie 2.0 project was described as "providing project oversight and evangelism to the academic community" (Visel, 2008). The choice of the word "evangelism" is telling in illuminating the heart of the purpose of this project. Sophie was promoting a certain vision of the future. Sophie embodies the values of free and open source, multimedia document creation, and new forms of authorship and intellectual property that the creators believed were going to be the future.

Unfortunately, they did not create a product that worked in its present time. It is not the job of librarians and digital humanists to use software we hope will work because it aligns with values we find important, it is our job to recommend and contribute to digital tools that won't eat our users' homework.

Although information about Sophie 2.0 and the developer site can still be accessed today, there is little word of what happened to the project and its history. You basically have to call Dan Visel and ask what happened. Scalar is probably the closest thing we have to a platform for creating nonlinear online books where users can incorporate and annotate multimedia content, or what USC and the Institute for the Future of the Book had hoped to accomplish with Sophie 2.0. Although everyone I talked to insisted that these platforms were not at all related and have different histories, Scalar also received funding from the Mellon Foundation and is based at the University of Southern California. Tara McPherson, who edited the volume that the "A Pedagogy for Original Synners," the article that argues for releasing incomplete user-unfriendly open source software, is currently a PI for Scalar. While Scalar is much better to use on many aspects, such as being designed around copyright and having very helpful tech support, I worry about the sustainability and digital preservation issues with this software, especially as more of us use it for scholarly purposes. Moreover, as a user of open source projects for digital humanities, I'm all too familiar with the issues of buggy software and lost work. While certainly not as extreme as in the case of Sophie 1.0 and Sophie 2.0, when paired with not publicly discussing

12/16 Kirby, Jasmine S. (2018) How NOT to create a digital media scholarship platform: the history of the Sophie 2.0 project, IASSIST Quarterly 42 (4), pp. 1-16. DOI: https://doi.org/10.29173/iq926 
the issues that led to Sophie 2.0's failure, makes me question what, if anything, we as digital humanists have learned from our mistakes.

\section{Takeaways, questions to ask when evaluating a digital scholarship tool}

- Has this been updated? When was the most recent update? Are updates regular or sporadic?

- How is this project funded? Is this grant funded? What happened to other recipients of these grants?

- How well does the software work? Has the software ever worked? Is this software supposed to work?

- Where can I get tech support? How fast is the response for questions about glitches? Are users told to fix glitches themselves?

- Was this software created accounting for intellectual property laws and other legal issues faced by users?

- Does this software claim to be meant for nontechnical users? Is there documentation? Is there a glossary for software-specific terms?

- Are there reviews? Demo projects? Are demo projects created by ordinary users or institutional groups with advanced IT resources?

\section{References}

Anderson, S. and Balsamo, A. (2008), “A Pedagogy for Original Synners”, in McPherson, T. (Ed.), Digital Youth, Innovation, and the Unexpected, MIT Press, Cambridge, Mass, pp. 241-259.

ArtsJ09. (2009), Sophie Presented by Holly Willis, available at: https://www.youtube.com/watch?v=KLTcdffTigA (accessed 16 March 2018).

Ballatore, A. and Natale, S. (2016), "E-readers and the death of the book: Or, new media and the myth of the disappearing medium", New Media \& Society, Vol. 18 No. 10, pp. 2379-2394.

Barnett, T. (2015), "Platforms for Social Reading: The Material Book's Return", Scholarly \& Research Communication, Vol. 6 No. 4, pp. 1-23.

Bjork, O. (2012), "Digital Humanities and the First-Year Writing Course", in Hirsch, B.D. (Ed.), Digital Humanities Pedagogy: Practices, Principles and Politics, Open Book Publishers, Cambridge, UK, pp. 97-119.

Bridle, J. (2007), "Sophie's Choice (a partial review)", Booktwo, 10 April, available at: http://booktwo.org/note-book/sophies-choice-a-partial-review/ (accessed 15 March 2018).

Dean, M.D. (2016), "A call to embrace social reading in higher education", Innovations in Education \& Teaching International, Vol. 53 No. 3, pp. 296-305.

Dombrowski, Q. (2014), “What Ever Happened to Project Bamboo?”, Literary and Linguistic Computing, Vol. 29 No. 3, pp. 326-339. 
Fuchs, I.H. (2008), "Challenges and Opportunities of Open Source in Higher Education", in Katz, R.N. (Ed.), The Tower and the Cloud: Higher Education in the Age of Cloud Computing, Educause, Boulder, CO, pp. 150-157.

Gaitán, S.B. (2011), “Children of the Screen: Teaching Spanish with Commentpress", Learning Through Digital Media, pp. 47-55.

Gold, M.K. (2011), "'Becoming Book-Like: Bob Stein and the Future of the Book' (Interview)", Kairos, Vol. 15 No. 2, available at: http://technorhetoric.net/15.2/interviews/ (accessed 11 March 2018).

Good, K.D. (2017), "Listening to Pictures: Converging media histories and the multimedia newspaper", Journalism Studies, Vol. 18 No. 6, pp. 691-709.

Herther, N.K. (2017), "Top Tools for Digital Humanities Research", Computers in Libraries, February, Vol. 37 No. 1, p. 28.

Hirschfeld, R., Haupt, M., Rüger, M., Brünn, P., Esterluß, R., Holz, N., Knebel, K., et al. (2008),

"SophieServer: The Future of Reading", Proceedings of the Sixth International Conference on Creating, Connecting and Collaborating Through Computing (C5 2008), IEEE Computer Society, Washington, DC, USA, pp. 29-35.

Holz, N., Hirschfeld, R., Lincke, J., Haupt, M. and Rüger, M. (2009), "Sophie Tools and Materials in Multimedia Book Creation", Proceedings of the 2009 Seventh International Conference on Creating, Connecting and Collaborating Through Computing, IEEE Computer Society, Washington, DC, USA, pp. 20-26.

"How To Kill an Open Source Project With New Funding". (2008), Slashdot, 3 October, available at: https://ask.slashdot.org/story/08/10/03/1547256/slashdot.sourceforge.net (accessed 6 February 2018).

Hudson, R. (2008), Sophie, Research Report, Transliteracies: Research in the Technological, Social, and Cultural Practices of Online Reading, available at:

http://transliteracies.english.ucsb.edu/post/research-project/researchclearinghouseindividual/sophie-2 (accessed 6 February 2018).

Koychev, I., Dicheva, D. and Nikolov, R. (2013), "Smartbook: Semantics Inside", Serdica Journal of Computing, Vol. 4 No. 2, pp. 263-278.

Lanier, J. (2010), You Are Not a Gadget: A Manifesto, 1st ed., Alfred A. Knopf, New York.

"Ipandeff". (2009), "DEVELOPMENT_OVERVIEW - Sophie 2.0", Wiki:DEVELOPMENT_OVERVIEW, 15 October, available at: http://sophie2.org/trac/wiki/DEVELOPMENT OVERVIEW (accessed 19 March 2018).

Morozov, E. (2013), To Save Everything, Click Here: The Folly of Technological Solutionism, First edition, Public Affairs, New York.

14/16 Kirby, Jasmine S. (2018) How NOT to create a digital media scholarship platform: the history of the Sophie 2.0 project, IASSIST Quarterly 42 (4), pp. 1-16. DOI: https://doi.org/10.29173/iq926 
Moyer, S. (2009), "What IF?” Humanities, August, Vol. 30 No. 4, available at:

https://www.neh.gov/humanities/2009/julyaugust/feature/what-if (accessed 8 March 2018).

Ochoa, X., Casali, A., Deco, C., Gerling, V., Frango, I., Fager, J., Carrillo, G., et al. (2013), “Analysis of Existing Technological Platforms for the Collaborative Production of Open Textbooks", presented at the EdMedia: World Conference on Educational Media and Technology, Association for the Advancement of Computing in Education (AACE), pp. 1106-1115.

Rich, M. (2009), "Curling Up With Hybrid Books, Videos Included", The New York Times, 1 October, p. $\mathrm{A} 1(\mathrm{~L})$.

Rüger, M., Stein, B. and Visel, D. (2008), "Sophie - The Future of Reading", Proceedings of the Sixth International Conference on Creating, Connecting and Collaborating Through Computing (C5 2008), IEEE Computer Society, Washington, DC, USA, pp. 13-20.

"Sophie": (2015), 19 June, available at:

https://web.archive.org/web/20150619042103/http://www.sophieproject.org/ (accessed 19 March 2018).

Stein, B. (2008), "The Evolution of Reading and Writing in the Networked Era", transcribed by Creech, A. The Serials Librarian, Vol. 54 No. 1-2, pp. 43-55.

Stein, B. and Visel, D. (2010), "Mao, King Kong, and the Future of the Book", Triple Canopy, No. 9, available at:

https://www.canopycanopycanopy.com/contents/mao king kong and the future of th e book (accessed 11 March 2018).

Stein, R. and Visel, D. (2007), "Sophie and the Future of Reading and Writing", Forum Futures 2007, presented at the 2006 Aspen Symposium, Forum for the Future of Higher Education, Cambridge, Mass, pp. 57-60.

Virshup, A. (1996), "The Teachings of Bob Stein", WIRED, 1 July, available at: https://www.wired.com/1996/07/stein/ (accessed 13 March 2018).

Visel, D. (2008), “AboutPage - Sophie 2.0", 15 December, available at: http://sophie2.org/trac/wiki/AboutPage (accessed 15 March 2018). Visel, D. (2018), Interview with author, 7 February.

\section{End-notes}

${ }^{1}$ Jasmine S. Kirby is a subject liaison librarian for psychology and human development and family studies at the lowa State University of Science and Technology in Ames, lowa.

15/16 Kirby, Jasmine S. (2018) How NOT to create a digital media scholarship platform: the history of the Sophie 2.0 project, IASSIST Quarterly 42 (4), pp. 1-16. DOI: https://doi.org/10.29173/iq926 
${ }^{2}$ Interview with Sophie user who wished to remain anonymous conducted March 1, 2018

${ }^{3}$ Institute for the Future of the Book

${ }^{4}$ Interview with Sophie user who wished to remain anonymous conducted March 1, 2018

${ }^{5}$ Interview with Sophie user who wished to remain anonymous conducted March 1, 2018.

${ }^{6}$ DRM is an acronym for Digital Rights Management

16/16 Kirby, Jasmine S. (2018) How NOT to create a digital media scholarship platform: the history of the Sophie 2.0 project, IASSIST 